\title{
ALTERNATIVE ALGORITHMS FOR COMPUTATIONAL FLUID DYNAMICS. FINAL REPORT
}

\author{
Anthony J.C. Ladd
}

March 1995

This is an informal report intended primarily for internal or limited external distribution. The opinions and conclusions stated are those of the author and may or may not be those of the Laboratory.

Work performed under the auspices of the Department of Energy by the Lawrence Livermore National Laboratory under Contract W-7405-Eng-48. 
This document was prepared as an account of work sponsored by an agency of the United States Covernment. Neither the United States Covernment nor the University of California nor any of their employees, makes any warranty, express or implied, or assumes any legal liability or responsibility for the accuracy, completeness, or usefulness of any information apparatus, product, or process disclosed, or represents that its use would not infringe privately owned rights. Reference herein to any specific commercial product, process, or service by trade name, trademark, manufacturer, or otherwise, does not necessarily constitute or imply its endorsement, recommendation, or favoring by the United States Covernment or the University of Califormia. The views and opinions of authors expressed herein do not necessarily state or reflect those of the United States Govemment or the University of Califomia, and shall not be used for advertising or product endorsement purposes.

This report has been reproduced directly from the best available copy.

Available to DOE and DOE contractors from the Office of Scientific and Technical Information

P.O. Box 62, Oak Ridge, TN 37831

Prices available from (615) 576-8401, FTS 626-8401

Available to the public from the

National Technical Information Service

U.S. Department of Commerce 5285 Port Royal Rd,

Springfield, VA 22161 


\section{DISCLAIMER}

Portions of this document may be illegible in electronic image products. Images are produced from the best available original document. 


\title{
Alternative Algorithms for Computational Fluid Dynamics. Final Report
}

\author{
Anthony J.C. Ladd \\ Theoretical and Applied Mechanics \\ Cornell University, Ithaca, New York 14859.
}

March 3, 1995

\begin{abstract}
Fluid tow is conventionally modeled by finite difference or finite element approximations to the Navier-Stokes equations. The key problem in such calculations is devising an efficient computational mesh on which to solve the equations; if the geometry is complex, extensive human intervention is usually necessary. Thus these methods are unsuitable for problems such as the motion of solid particulates in suspension, where there may be many thousands of objects whose positions are constantly varying over the course of the simulation. Over the past few years I have developed an alternative strategy for modeling solid-fluid flows, based on a discrete Boltzmann model, in which the particle velocities are sampled from a small well-chosen set, commensurate with the underlying spatial lattice. This leads to a simple and fast numerical algorithm which can solve fluid flow problems with high accuracy on relatively crude spatial meshes. Thus it has been possible to track the motion of around 1000 hydrodynamically interacting particles on a desktop workstation. A preliminary account of some of this work was published in Physical Review Letters [1]; a complete account of the method is given in two papers published by the Journal of Fluid Mechanics [2,3].
\end{abstract}




\section{Computational method}

The numerical simulations in this work are based on a combination of molecular dynamics, which is used to follow the dynamics of the solid particles, and a lattice-Boltzmann model of the background tluid phase. This computational scheme is remarkably effective in reproducing the hydrodynamic forces and torques between the suspended solid particles, both in the creeping-flow $\left(R_{e}=0\right)$ regime and at finite Reynolds number. A detailed discussion of the method, together with extensive numerical tests, has already been reported $[2,3]$; we give a brief summary below.

In the lattice-Boltzmann approximation, the fundamental quantity is the discretized one-particle velocity distribution function $n_{i}(\mathbf{r}, t)$, which describes the number of particles at a particular node of the lattice $\mathbf{r}$, at a time $t$, with a velocity $\mathbf{c}_{i} ; \mathbf{r}, t$, and $\mathbf{c}_{i}$ are discrete, whereas $n_{i}$ is continuous. The hydrodynamic fields, mass density $\rho$, momentum density $j$, and momentum flux $\Pi$, are moments of this discrete velocity distribution:

$$
\rho=\sum_{i} n_{i}, \quad \mathbf{j}=\sum_{i} n_{i} \mathbf{c}_{i}, \quad \boldsymbol{\Pi}=\sum_{i} n_{i} \mathbf{c}_{i} \mathbf{c}_{i}
$$

The computational utility of the lattice-Boltzmann equation is related to the realization that only a small set of discrete velocities are necessary to simulate the Navier-Stokes equations [4]. The specific model used in this work has 18 different velocities corresponding to the nearneighbor and second-neighbor directions of a simple cubic lattice. Thus there are six velocities of speed 1 , corresponding to (100) directions in the lattice and 12 velocities of speed $\sqrt{2}$, corresponding to the (110) directions, for a total of 18 . Note that the velocities are such that all particles move from node to node simultaneously.

The time evolution of the distribution functions $n_{i}$ is described by a discrete analogue of the Boltzmann equation ([2]),

$$
n_{i}\left(\mathbf{r}+\mathbf{c}_{i}, t+1\right)=n_{i}(\mathbf{r}, t)+\Delta_{i}(\mathbf{r}, t),
$$

where $\Delta_{i}$ is the change in $n_{i}$ due to instantaneous molecular collisions at the lattice nodes. The post-collision distribution $n_{i}+\Delta_{i}$ is propagated for one time step, in the direction $c_{i}$. The collision operator $\Delta(n)$ depends on all the $n_{i}$ 's at the node, denoted collectively by $n(r ; t)$; it can take any form, subject to the constraints of mass and momentum conservation [2]. Note that the evolution equation is a discretization of a linear partial differential equation; the non-linear term in the momentum equation comes from averaging the linear pde's over the different velocity directions. 
The solid particles are defined by a boundary surface, of arbitrary size and shape, which cuts some of the links between lattice nodes The fluid particles moving along these links interact with the solid surface at boundary nodes placed halfway along the links. Thus a discrete representation of the particle surface is obtained, which becomes more and more precise as the particle gets larger. At each boundary node there are two incoming distributions. By exchanging population density between these two distributions, the local momentum density can be modified to match the velocity of the solid particle surface at the boundary node, without affecting either the local mass density or the local fluid stress. As a result of the boundary-node interactions, forces are exerted on the solid particles; thus momentum is exchanged locally between the fluid and the solid particle, but the combined momentum of solid and fluid is conserved. The forces and torques on the solid particle, obtained by summing the individual point forces over all the boundary nodes associated with a particular particle, are then used to update the particle velocity and angular velocity, according to the laws of Newtonian mechanics.

There are two major alternatives to the lattice-Boltzmann scheme for simulating particle suspensions; boundary-integral methods $[5,6]$ and finite-difference/finite-element methods. The primary drawback of boundary integrals is that they generate large linear systems of equations. Thus the computational cost of these schemes scales as $N^{2}$ or $N^{3}$ ( $N$ is the number of particles) and they are unsuitable for simulations of more than a few hundred spheres; the lattice-Boltzmann algorithm models local time-dependent hydrodynamic interactions and thus the computational cost scales linearly with system size. Moreover boundary-integral methods are only valid in the creeping-flow regime $\left(R_{e}=0\right)$. Thus they are unsuitable for both types of problem we wish to solve. Finite-difference methods are not usually applied to multiparticle suspensions; the unresolved difficulty is how to determine a suitable mesh for a large number of objects, particularly when the boundary surfaces are moving in time. It is possible to construct a finite-difference scheme on a regular mesh, similar to that used in the lattice-Boltzmann method, but the accuracy is much less for comparable size meshes [3]. Thus the lattice-Boltzmann method is the only viable computational approach, at present, to the problems we wish to solve. Moreover it is a highly parallel algorithm, with little global communication necessary; thus it is ideal for a distributed memory computer such as the Meiko CS2 and the IBM SP2. Parallel versions of the programs have been developed during this project (see below) and are running on the Meiko CS2 at Lawrence Livermore National Laboratory. 


\section{Work accomplished in FY94}

\subsection{Structure and dynamics of sedimenting sus- pensions}

The sedimentation of a suspension of rigid non-interacting spheres can be characterized by the volume fraction occupied by the solid particles, the Reynolds number $R_{e}=2 U a \rho / \eta$, and the Peclet number $P_{e}=U a / D$. In these expressions $U$ is the average sedimentation velocity (fall speed), $a$ is the particle radius, $\rho$ is the mass density of the fluid, $\eta$ is the viscosity, and $D$ is the diffusion coefficient of the solid particles. In these studies we are interested in the lowReynolds number, high-Peclet limit, where there is no fluid inertia and no Brownian motion of the solid particles; typical particle sizes in laboratory experiments range from 10-1000 microns. In such cases, the microstructure, that is the relative arrangements of the solid particles, can be quite different from that of equilibrium suspensions, which is the well-known thermodynamic distribution of rigid spheres. We are using computer simulations to study the connection between suspension microstructure and fluctuations in particle velocity, which leads to hydrodynamically induced dispersion of the solid particles, and in some instances to instabilities in the sedimenting suspension. To date, there has been no experimental attempts to study the microstructure of sedimenting suspensions, although it might be possible to do so by light-scattering. Our major computational effort in this area is directed towards a study of sedimentation of monodisperse suspensions. Our primary goal is to understand the connection between suspension microstructure and particle velocity fluctuations. For a monodisperse suspension of spheres characterized by the thermodynamic equilibrium distribution, a relatively simple calculation shows that fluctuations in particle velocity diverge with the linear size of the container [7]. The velocity disturbance at a point due to a sphere some large distance $R$ away is proportional to $1 / R$; thus a distant sphere induces fluctuations in particle velocity of order $1 / R^{2}$. On integration over volume, this leads to a divergence in the velocity fluctuations. However, recent experimental studies $[8]$ indicate that fluctuations in particle velocity are independent of system size. A theoretical explanation of this has already been put forward [9]; the authors argue that changes in the suspension microstructure during sedimentation lead to a screening of the hydrodynamic interactions and therefore to finite velocity fluctuations. The mechanism is analogous to the Debye-Huckel screening in ionic solutions. In earlier simulations we attempted to observe the microstructural changes predicted by this theory [10], but were unable to do so. Unfortunately, the computational method used for these sim- 
ulations precluded the use of very large systems (no more than 108 spheres) and thus our results were inconclusive. We now have a new computational method which is able to simulate systems of up to $10^{5}$ spheres on present parallel supercomputers. These are large enough samples to answer the question as to whether the absence of a divergence in the velocity fluctuations is due to microstructural changes in the bulk suspensions, as suggested by Koch and Shaqfeh [9], or to some other cause, such as container walls or inertia $[11,12]$. We emphasize that, although there are several experimental studies of fluctuations in sedimenting suspensions $[8,13,14,15]$, none of these experiments can characterize the suspension microstructure.

Because of the reduced funding level in FY94 ( $\$ 80 \mathrm{~K}$ versus a request of about $\$ 250 \mathrm{~K}$ ) the level of effort has been much less than initially proposed. Nevertheless, significant progress has been made towards completing the two application milestones described in the original proposal. The main focus this year has been to develop a code that can track the dynamical motion of spheres in suspension. Previous versions of the code could only simulate objects that were fixed in space. The work has been directed towards studying the motion of suspensions of sedimenting spheres and answering the questions discussed above.

Test calculations for the sedimentation of small numbers of spheres have been compared with earlier results using different methods (suitable only for small numbers of particles), to validate the simulations. Video imaging of the simulation data for up to eight thousand spheres shows that large scale structures are generated during sedimentation; these will be studied in detail in the near future, by the parallel codes developed under this proposal.

A parallel version of the code able to simulate tens of thousands of spheres has been prepared for the Meiko. It is a unique tool for probing the microstructural changes that take place during the flow of particulates. Unfortuately, because of hardware problems with the Meiko in its preacceptance configuration, it was not possible to complete definitive calculations during the lifetime of the proposal. During my sabbatical year at Cornell, I am continuing these investigations, initially on the Meiko, but later on the IBM SP2. A proposal for a substantial quantity of supercomputer time has been submitted.

Investigations into possible technical improvements to the code, that may improve its perfomance substantially, were begun during a visit to ISCR by Oliver Behrend. In the last few months I have made substantial revisions to the code which improve both the accuracy and the efficiency of dynamical simulations. 


\section{Other applications}

These new algorithms are extremely flexible, and can be applied to many areas involving fluid flow in complex geometries. Recent applications include the simulation of fluid flow through filters composed of fine wire fibres. Our lattice-Boltzmann simulations are of comparable accuracy to standard commercial packages, such as FIDAP, and are much faster to implement. These codes are currently in use by the hazards control group at LLNL (Bob Corey) Moreover, no other codes available today have the ability to track the motion of large numbers of suspended particles. Typical future problems to be studied involve the rheological and transport properties of particle suspensions and slurries. Areas of application include critical technology areas such as precision coating, as well as more mundane but industrially important applications such as solids transport.

A slightly modified version of the code allows us to study the dynamics of suspensions of colloidal particles, moving under the influence of hydrodynamic and Brownian forces. The simulation method has been use to help intepret results of light-scattering experiments $[1,16]$.

\section{Publications}

I published two papers describing the general method in $1994[2,3]$; they were submitted before the beginning of this work. The preliminary account of work on colloidal suspensions (JC-UCRL 116648) was published very recently in Physical Review Letters [16]. A more complete account will be submitted soon. The sedimentation simulations are still in progress; I have reported preliminary results at a conference in Manchester, England in July 1994.

\section{References}

(1) A. J. C. Ladd. Short-time motion of colloidal particles: Numerical simulation via a fluctuating lattice-Boltzmann equation. Phys. Rev. Lett., 70:1339, 1993.

[2] A. J. C. Ladd. Numerical simulations of particulate suspensions via a discretized Boltzmann equation Part I. Theoretical foundation. J. Fluid Mech., 271:285, 1994.

[3] A. J. C. Ladd. Numerical simulations of particulate suspensions via a discretized Boltzmann equation Part II. Numerical results. J. Fluid Mech., 271:311, 1994.

[4] U. Frisch, B. Hasslacher, and Y. Pomeau. Lattice gas automata for the Navier-Stokes equation. Phys. Rev. Lett., 56:1505, 1986. 
(5) J. F. Brady and G. Bossis. Stokesian dynamics. Ann. Rev. Fluid. Mech., 20:111, 1988.

6) S. J. Karrila, Y. O. Fuentes, and S. Kim. Parallel computational strategies for hydrodynamic interactions between rigid particles of arbitrary shape in a viscous fluid. J. Rheology, 33:913, 1989.

[7] R. E. Caflisch and J. H. C. Luke. Variance in the sedimentation speed of a suspension. Phys. Fluids, 28:759, 1985.

[8] H. Nicolai and E. Guazzelli. Effect of the vessel size on the hydrodynamic diffusion of sedimenting spheres. Phys. Fluids, 7:3, 1995.

[9] D. L. Koch and E. S. G. Shaqfeh. Screening in sedimenting suspensions. J. Fluid Mech., 224:275, 1991.

(10) A. J. C. Ladd. Dynamical simulations of sedimenting spheres. Phys. Fluids A, 5:299, 1993.

[11] E. J. Hinch. Sedimentation of small particles. In E. Guyon, J. P. Nadal, and Y. Pomeau, editors, Disorder and Mixing, Dordrecht, 1985. Kluwer Academic.

[12] D. L. Koch. Hydrodynamic diffusion in dilute sedimenting suspensions at moderate Reynolds numbers. Phys. Fluids A, 5:1141, 1993.

[13] J. M. Ham and G. M. Homsy. Hindered settling and hydrodynamic dispersion in quiescent sedimenting suspensions. Int. $J$. Multiphase Flow, 14:533, 1988.

[14] J.-Z. Xue, E. Herbolzheimer, M. A. Rutgers, W. B. Russel, and P. M. Chaiken. Diffusion, dispersion, and settling of hard spheres. Phys. Rev. Lett., 69:1715, 1992.

(15) H. Nicolai, B. Herzhaft, E. J. Hinch, L Oger, and E. Guazzelli. Particle velocity fluctuations and hydrodynamic self-diffusion of sedimenting non-Brownian spheres. Phys. Flusds, 7:12, 1995.

[16] A. J. C. Ladd and H. Gang and J. X. Zhu and D. A. Weitz. Time-dependent collective diffusion of colloidal particles. Phys. Rev. Lett., 74:318, 1995. 